\title{
Dispersion Engineered Silicon Nanocrystal Slot Waveguides for Soliton Ultrafast Optical Processing
}

\author{
Francesco De Leonardis ${ }^{1}$ and Vittorio M. N. Passaro ${ }^{2}$ \\ ${ }^{1}$ Photonics Research Group, Dipartimento di Ingegneria dell'Ambiente e per lo Sviluppo Sostenibile, Politecnico di Bari, \\ viale del Turismo n. 8, 74100 Taranto, Italy \\ ${ }^{2}$ Photonics Research Group, Dipartimento di Elettrotecnica ed Elettronica, Politecnico di Bari, via Edoardo Orabona n. 4 , \\ 70125 Bari, Italy \\ Correspondence should be addressed to Vittorio M. N. Passaro, passaro@deemail.poliba.it
}

Received 28 April 2011; Accepted 16 June 2011

Academic Editor: Anthony Kenyon

Copyright ( 2011 F. De Leonardis and V. M. N. Passaro. This is an open access article distributed under the Creative Commons Attribution License, which permits unrestricted use, distribution, and reproduction in any medium, provided the original work is properly cited.

We propose, for the first time to our knowledge, the theoretical investigation of silicon nanocrystals-based sandwiched slot waveguides which are dispersion-engineered for exciting optical solitons inside very short structures (only $1.2 \mathrm{~mm}$ long). Several parametric simulations have been performed by means of finite element method in order to individuate the best waveguide crosssections for achieving an anomalous dispersion regime around $1550 \mathrm{~nm}$.

\section{Introduction}

In the last few years, silicon has become the ideal platform for integrated optics and optoelectronics due to its broad application potential, going from optical interconnects to biosensing [1]. The quality of commercial silicon wafers driven by microelectronics industry still continues to improve while the cost continues to decrease. Moreover, the compatibility with silicon integrated circuits manufacturing and silicon micro-electromechanical systems (MEMSs) technology is very high, and it represents another important reason for this interest in silicon photonics [2,3]. Recently, several high-speed electro-optical functionalities have been demonstrated in silicon, such as a $40 \mathrm{Gbit} / \mathrm{s}$ modulator based on free-carrier (FC) plasma dispersion effect [4]. However, in order to reach much higher speeds (100 Gbit/s and beyond), the electrical domain should be completely overcome and the information data transfer should be entirely processed in the optical domain (ultrafast alloptical processing). In this sense, a number of research study has been performed in order to exploit the ultrafast third-order nonlinear effects in silicon. As a transmission medium, silicon has much higher nonlinear effects than the commonly used silicon dioxide (i.e., optical fibers), in particular Kerr and Raman effects. Additionally, silicon-oninsulator (SOI) waveguides can confine the optical field to an area that is approximately 100 times smaller than the modal area in a standard single-mode optical fiber. Consequently, it is expected that nonlinear optical effects should occur in these waveguides at lower input powers, similar to those used in optical communications systems. For example, several recent experimental and theoretical studies have been focused for exploiting the stimulated Raman scattering (SRS) in the SOI integrated platform. In fact, the need for active devices in SOI has stimulated an increasing research effort in Raman-based light amplification [5-12] and generation [13-20]. Moreover, the ability to generate and utilize large bandwidths using parametric processes is crucial for a large range of photonic applications, including ultrafast signal processing and telecommunications [2124]. In addition to the large number of above-mentioned nonlinear applications, another very important nonlinear phenomenon is the formation of optical solitons. Solitons have been observed inside silica fibers, and they have found a large number of applications, ranging from optical switching to supercontinuum generation [25]. Thus, the possibility 
to generate solitons inside SOI waveguides could favour a number of very promising applications for ultrafast optical signal processing and fast soliton telecommunications systems. Recently, solitons have been observed for the first time inside a $5 \mathrm{~mm}$ long SOI waveguide by excitation with a $120 \mathrm{fs}$ input Gaussian pulse, aligned as a quasi-TM mode [26]. However, the enhancement of nonlinear effects in siliconbased photonic components is still a key point in order to achieve active and more complex guiding structures. Novel nonlinear materials, such as silicon nanocrystals (Si-nc), can be developed by using conventional CMOS fabrication processes. In particular, this material possesses interesting optical properties, that is, nonlinear Kerr coefficient $\left(n_{2}\right)$ one or two orders of magnitude larger than in silicon [2729]. In this paper, for the first time to our knowledge, the formation of optical solitons is theoretically demonstrated in appropriately engineered SOI slot waveguides based on Sinc.

In Section 2, a number of parametric simulations based on the finite element method have been carried out to find efficient guidelines for the design of optimal Si-nc slot waveguides able to induce the soliton formation over a millimetre scale length. In addition, some comparisons with standard SOI rib waveguides are shown with the aim to well outline the great potential of Si-nc technology. In Section 3, a semianalytical model is presented in order to investigate the space-time evolution of Gaussian pulses into dispersion engineered Si-nc slot structures for exciting ultrafast optical solitons. We introduce in this section a number of effective physical parameters in order to describe the soliton excitation in a engineering fashion, taking into account the free carrier absorption (FCA) where the free carriers are generated mainly by two photon absorption (TPA) of the input pulse, and self-phase-modulation (SPM) effect as induced by the Kerr nonlinearity. We conjecture that the model used in this work could be a flexible tool for predicting the pulse evolution inside Si-nc slot waveguides with good accuracy, after an a priori setting of previously introduced effective parameters. Moreover, the proposed model represents a useful starting step to develop a more accurate and complete mathematical model, avoiding any a priori assumption. In the final part of this section, a number of simulations are presented to investigate the formation of optical solitons. Finally, Section 4 summarizes the conclusions.

\section{Waveguide Design}

In this section, a numerical investigation of group velocity dispersion (GVD) in Si-nc slot waveguides is presented. Only a few papers proposed in the literature have analyzed the optical dispersion properties for highly nonlinear slot waveguides. In particular, in the work proposed in [30], slot waveguides were designed in order to obtain flat and low dispersion behaviour over a wide wavelength range. To this aim, Si-nc and chalcogenide glass were considered as slot materials, achieving a best case of $0 \pm 160 \mathrm{ps} /(\mathrm{nm} \cdot \mathrm{km})$ over a 244-nm bandwidth. More recently, a silicon strip/slot hybrid waveguide was proposed [31] for inducing flattened dispersion of $0 \pm 16 \mathrm{ps} /(\mathrm{nm} \cdot \mathrm{km})$ over a $553-\mathrm{nm}$ wavelength

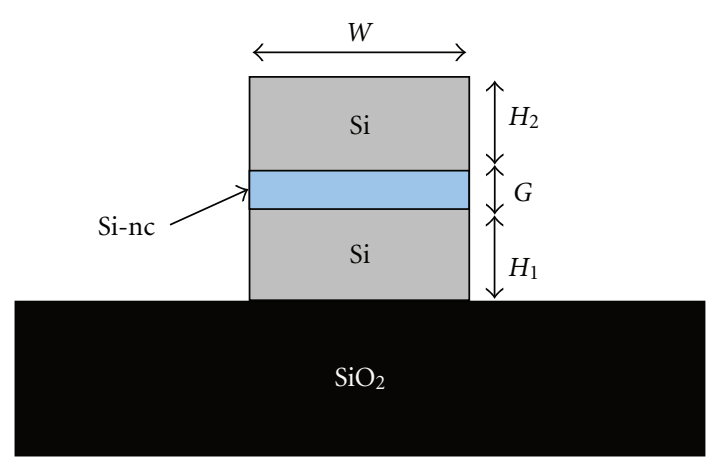

FIgURE 1: Cross section of SOI slot waveguide based on Si-nc.

range, that is, 20 times flatter than in previous results. Thus, this kind of waveguide exhibits flattened dispersion in the wavelength range from 1562-nm to 2115-nm, which is potentially useful for both telecom and mid-infrared applications. In spite of these very interesting results [30, 31], where the requirement was focused over flat and low dispersion, a completely different approach is followed in this section. In fact, a full parametric analysis is presented here in order to design the Si-nc slot waveguides with a desired GVD coefficient. In particular, the goal is to find the design guidelines to optimise the excitation of optical soliton in Si-nc slot waveguides. It is well known as optical solitons result from a critical interplay between simultaneous effects, group-velocity dispersion and self-phase modulation (SPM), when the wavelength of the exciting input optical pulse is placed inside the anomalous dispersion region for the specific guiding structure. The GVD-induced pulse broadening is known to scale with the dispersion length $L_{D}=T_{0}^{2} /\left|\beta_{2}\right|$, where $\beta_{2}$ is the GVD coefficient and $T_{0}$ is the pulse width, whereas the SPM-induced chirp scales with the nonlinear length $L_{\mathrm{NL}}=\left(\gamma P_{0}\right)^{-1}$, being $\gamma=$ $2 \pi n_{2} /\left(\lambda A_{\text {eff }}\right)$ the nonlinear parameter, $n_{2}$ the Kerr coefficient, and $P_{0}$ the peak power of pulses launched at wavelength $\lambda$ into the fundamental mode with effective mode area $A_{\text {eff. }}$. The formation of the fundamental soliton ideally requires $L_{\mathrm{NL}}=L_{D} \ll L$ for a waveguide of length $L$. The previous relationship indicates that both $\left|\beta_{2}\right|$ and $\gamma$ should be quite large to induce the formation of solitons in short waveguides, with lengths $L \leq 1 \mathrm{~cm}$. To this aim, the horizontal slot waveguide based on Si-nc and sketched in Figure 1 should guarantee an optimization in the soliton formation with respect to standard SOI rib waveguides. In the slot waveguide of Figure 1, the rib width is $W$, the slot region filled with Sinc has thickness $G$, and the bottom and top silicon layers have thickness $H_{1}$ and $H_{2}$, respectively. Thus, a number of geometrical parameters can be opportunely selected in order to induce values of $\left|\beta_{2}\right|$ inside the structure anomalous dispersion region as large as possible.

In addition, the waveguide in Figure 1 allows an electric field enhancement to be achieved in the slot region, where the Si-nc highly nonlinear material is present. This enhancement of vertical electric field component $E_{y}$ (quasi-TM mode) is given by a factor $\left(n_{\mathrm{Si}} / n_{\mathrm{Si}-\mathrm{nc}}\right)^{2} \cong 4.5$, being $n_{\mathrm{Si}}$ and $n_{\mathrm{Si}-\mathrm{nc}}$ the refractive index of silicon and Si-nc layers, respectively 


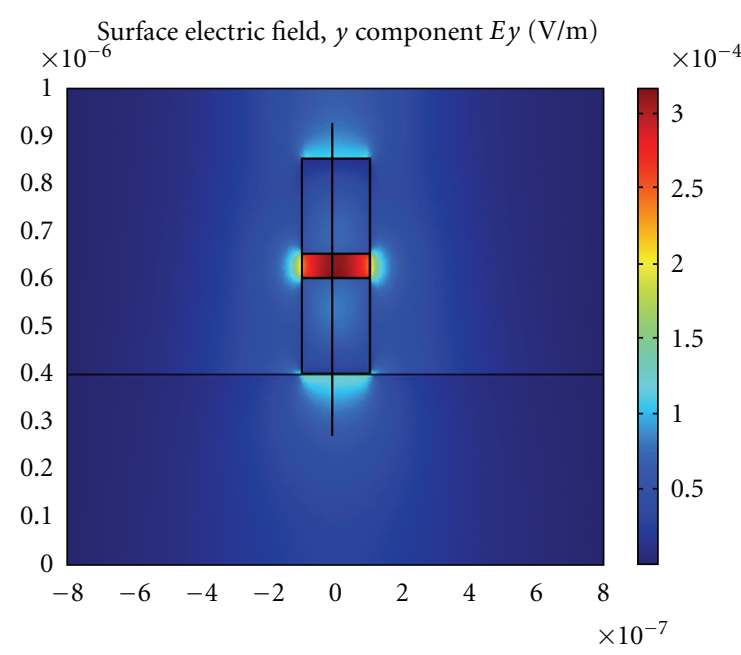

(a)

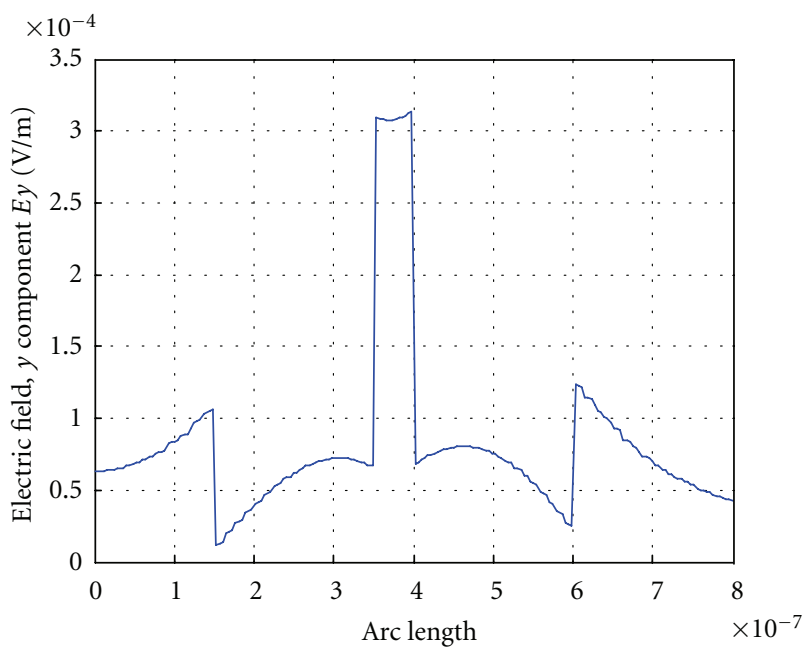

(b)

Figure 2: (a) 2D distribution of $E_{y}$ component; (b) Electric field profile along the line sketched in (a).

[27]. This effect is well evident in Figure 2, where the $E_{y}$ component is shown for a Si-nc slot waveguide operating at $\lambda=1550 \mathrm{~nm}$ with $H=H_{1}=H_{2}=200 \mathrm{~nm}, W=200 \mathrm{~nm}$ and $G=50 \mathrm{~nm}$.

In order to investigate the influence of Si-nc slot waveguide geometrical parameters on the optical dispersion properties, the following design variable is introduced:

$$
q=\frac{H}{W} \quad \text { with } H=H_{1}=H_{2} .
$$

In Figure 3 GVD coefficient spectra are shown for different values of $q$ parameter and slot thickness $G$. Each curve has been achieved by performing a number of simulations based on full vectorial finite element method (FEM) [32] in the wavelength range $1400-1700 \mathrm{~nm}$, in order to obtain the propagation constant spectra of quasi-TM modes. In all simulations, the Sellmeier equation has been used for index

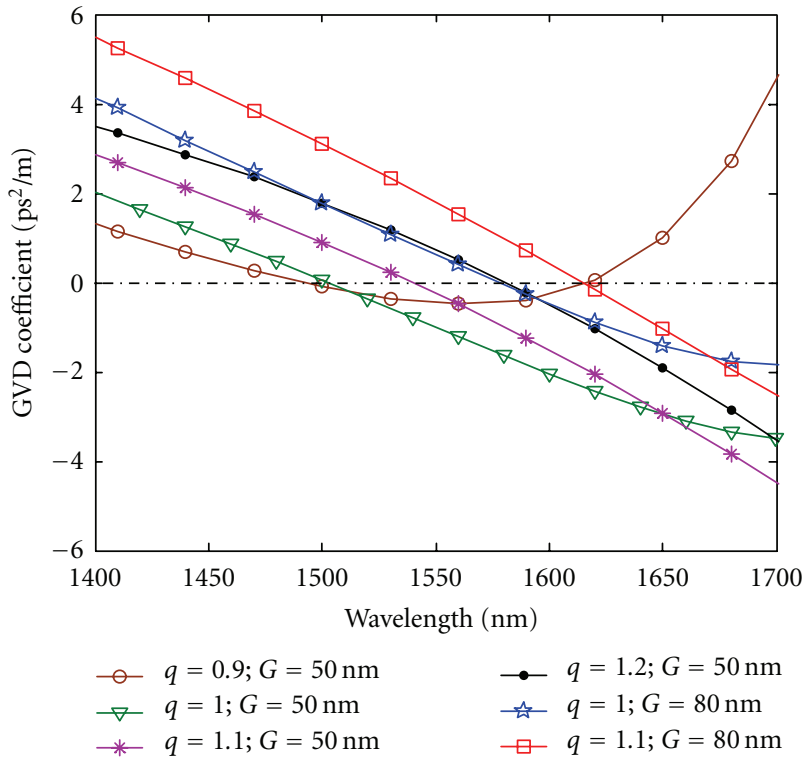

Figure 3: GVD coefficient versus wavelength for various $q$ and $G$.

dispersion. The differentiations from above-mentioned spectra lead us to calculate the GVD coefficient.

Several features of Figure 3 are noteworthy. For a given value of $G$, the zero GVD point (ZGVD) shifts towards higher wavelengths with increasing $q$ value. On the contrary, for a given value $q$, the ZGVD point can be shifted towards smaller wavelengths by decreasing the thickness $G$ of the slot region filled with Si-nc. Moreover, in the wavelength range 1400$1700 \mathrm{~nm}$, the GVD spectrum presents two ZGVD points (i.e., see black curve) for $G<80 \mathrm{~nm}$ and $q<1$. Therefore, this condition can open the possibility to design flat dispersion waveguides in the anomalous dispersion region. On the contrary, the GVD spectrum has only one ZGVD point in the same wavelength range for $q \geq 1$, inducing more and more negative values of $\beta_{2}$ (i.e., red and blue curves). Thus, it seems to be evident how appropriate combinations of $G$ and $q$ can give an optimization of soliton excitation conditions in very short waveguides. To this aim, a number of parametric simulations will be shown to find the best choice of geometrical slot waveguide parameters for generating optical solitons at $1550 \mathrm{~nm}$.

In Figure 4, the GVD coefficient is shown at $\lambda=1550 \mathrm{~nm}$ as a function of $G$ for different values of $q$ and $W$. The plots clearly show how $\beta_{2}$ coefficient monotonically decreases or increases as a function of $G$ for $q<1$ or $q \geq 1$, respectively.

It is worth to note the curve upshift or downshift with increasing $W$, depending on the specific value of $q$ parameter. For $q=0.5$ and $q=1.1, \beta_{2}$ coefficient for $W=240 \mathrm{~nm}$ is shifted towards higher values with respect to the case $W=200 \mathrm{~nm}$. The opposite trend can be observed for $q=0.7$. Finally, only two cases can induce anomalous dispersion region: (i) $W=200 \mathrm{~nm}$ and $q=0.5$ and (ii) $W=200 \mathrm{~nm}$ and $q=1.1$. The previous comments seem to indicate a nonlinearly predictable trend. In fact, it is clear that $q$ parameter plays a crucial rule in dispersion tailoring. In this sense, GVD coefficient is shown in Figure 5 versus 


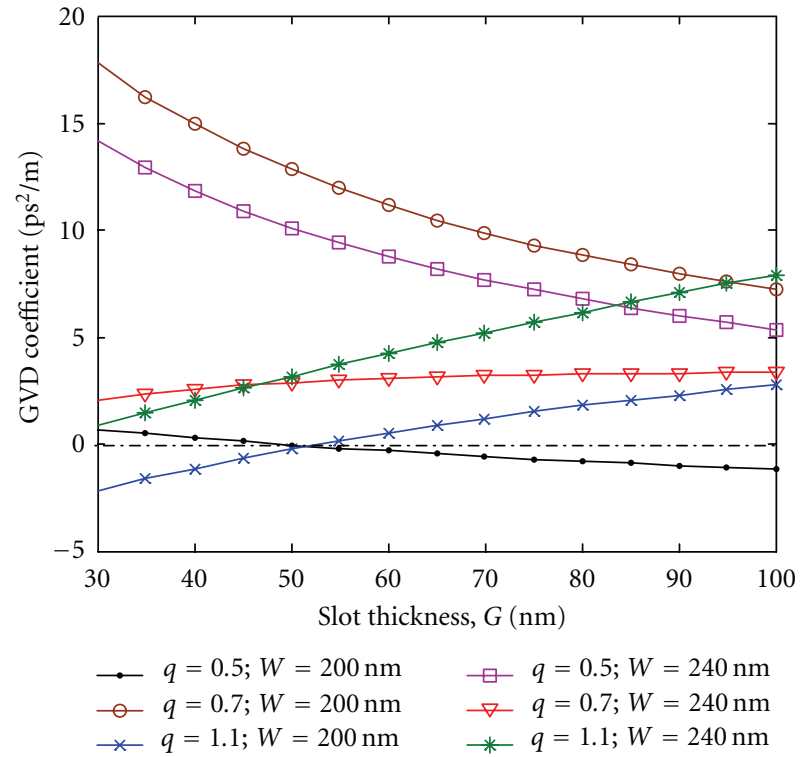

Figure 4: GVD coefficient versus $G$ for different values of $q$ and $W$ $(\lambda=1550 \mathrm{~nm})$.

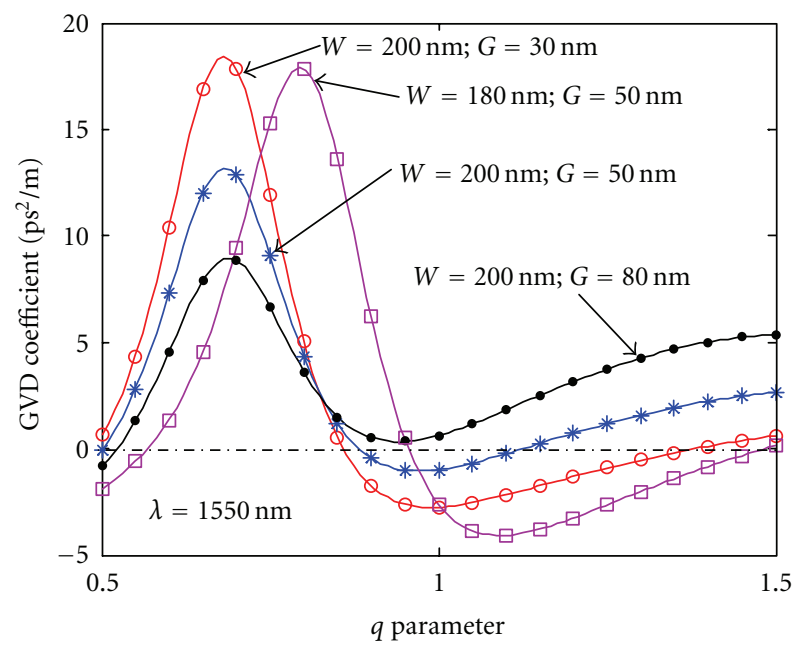

Figure 5: GVD coefficient versus $q$ for different values of $W$ and $G$ $(\lambda=1550 \mathrm{~nm})$.

$q$ for different values of $G$, assuming $W=200 \mathrm{~nm}$ and $\lambda=1550 \mathrm{~nm}$. Thus, this plot could be useful not only for best understanding the before described behavior, but also as a guideline to design the Si-nc slot waveguides characterized by a specific GVD coefficient.

The curves indicate how $\beta_{2}$ coefficient presents a positive peak, whose position is independent from the slot thickness $G$ but only on the rib width $W$. In fact, maxima points are obtained with $q \cong 0.68$ and $q \cong 0.79$ for $W=$ $200 \mathrm{~nm}$ and $180 \mathrm{~nm}$, respectively. Thus, Figure 5 shows a shift towards higher $q$ values with decreasing $W$. In addition, the positive peak is lower with increasing $G$ for a given $W$. Moreover, the curve shape presents an absolute minimum having negative or positive values for $G=30,50 \mathrm{~nm}$ and

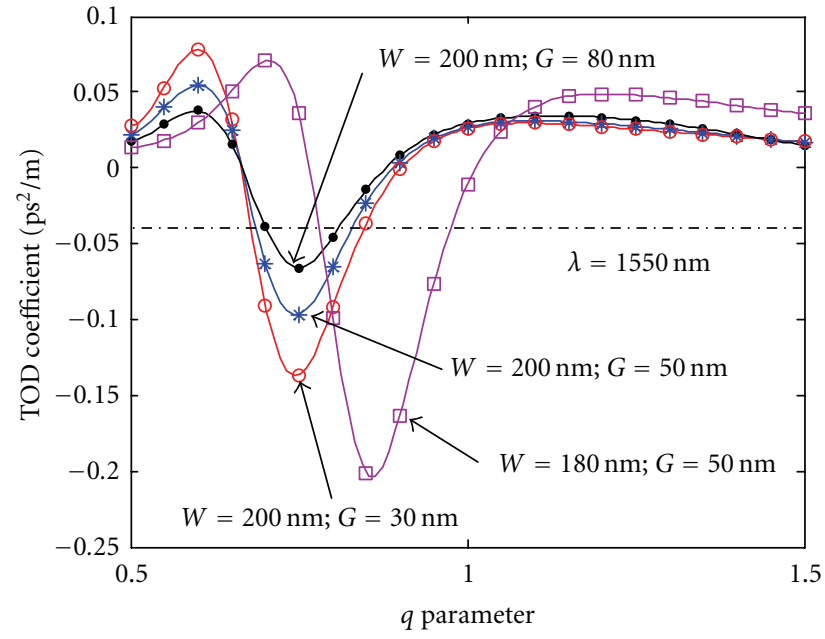

FIGURE 6: TOD coefficient versus $q$ for different values of $W$ and $G$ $(\lambda=1550 \mathrm{~nm})$.

$80 \mathrm{~nm}$, respectively, for $W=200 \mathrm{~nm}$. Thus, for $G<80 \mathrm{~nm}$ and $W=200 \mathrm{~nm}$, it is possible to find a range of $q$ values, where anomalous dispersion regime always occurs. It is worth to note that the before-mentioned range decreases with increasing $G$. Finally, it is important to outline that for a given value $G$, it is possible to increase the $q$ range, where anomalous dispersion regime occurs by decreasing the rib width and, at the same time, giving larger negative values of $\beta_{2}$. These unique features evidenced by curves of Figure 5 are of considerable importance. In fact, the previous plots indicate that the optimum value of $q$ must be selected around the minimum point in the $\beta_{2}$ curve shape, by this way achieving large negative values and, then, optimizing the soliton excitation.

Moreover, this kind of choice for $q$ allows an additional positive effect to occur. In fact, choosing $q$ around the minimum point of GVD, the third-order dispersion (TOD) $\beta_{3}$ coefficient will move around the zero point. This circumstance is evident in Figure 6, where TOD coefficient at $\lambda=1550 \mathrm{~nm}$ is shown as a function of $q$ for different values of $G$, assuming $W=200 \mathrm{~nm}$.

The condition zero-TOD represents a considerable advantage, since the pulse propagation, and, then, the soliton formation is not influenced by any detrimental effect induced by TOD. Thus, Si-nc slot waveguides offer the possibility to set an appropriate value of $q$ to simultaneously achieve large negative $\beta_{2}$ values as well as negligible $\beta_{3} \approx 0$. Therefore, these numerical investigations put into evidence this unique feature, that is, an additional potential of the Si-nc slot waveguides with respect to SOI rib waveguides. To better outline this aspect, we will also show the influence of SOI rib waveguide sizes on GVD coefficients. The attention has been focused on SOI waveguides with small cross-section, since it is also usually needed to have low effective recombination lifetimes, $\tau_{\text {eff, }}$ so reducing the detrimental effects induced by free carrier absorption (FCA) and two photon absorption (TPA) [12].

A submicron standard rib SOI structure $(G=0)$ with rib width $W=400 \mathrm{~nm}$ has been also investigated in this work. 


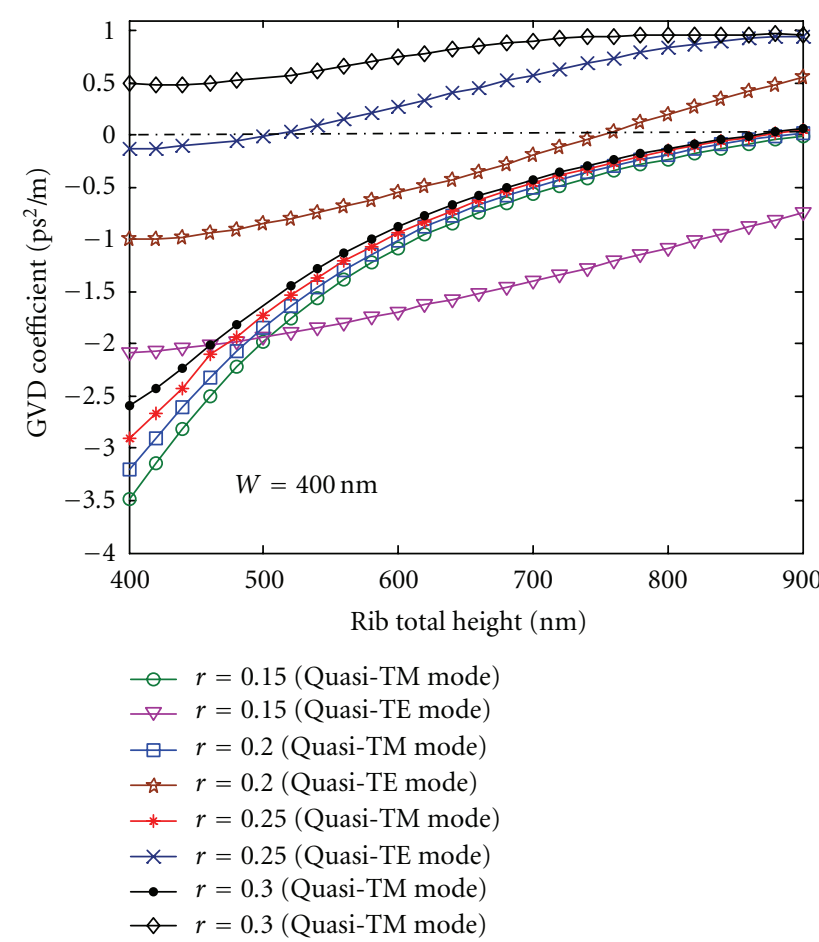

FIGURE 7: GVD coefficient versus rib total height for various $r$ and quasi-TE and quasi-TM modes in standard SOI rib waveguides $(\lambda=$ $1550 \mathrm{~nm})$.

A parametric plot of GVD coefficient $\beta_{2}$ versus rib total height $(H)$ is sketched in Figure 7 for different values of rib parameter $r=H_{s} / H$ (being $H_{s}$ the slab height) and for quasiTE and quasi-TM modes, assuming $\lambda=1550 \mathrm{~nm}$.

Figure 7 shows how quasi-TM modes have a GVD coefficient in the anomalous region for each considered value of $r$, with $H$ ranging from 400 to $900 \mathrm{~nm}$. Quite different is the behavior for quasi-TE modes. In fact, for $r=0.3$ and $r=0.15$, the GVD coefficient exclusively moves inside normal or anomalous dispersion region, respectively. On the contrary, for $0.15<r \leq 0.25 \beta_{2}$ coefficient overspreads both normal and anomalous region, reaching the zero dispersion condition for $H=749.6 \mathrm{~nm}$ and $505.5 \mathrm{~nm}$ with $r=0.2$ and $r=0.25$, respectively. It is worth noting that these ZGVD points move towards larger values of total rib height with decreasing $r$. As it is evident from the previous plots, the curves do not present any maximum or minimum point. Thus, differently from cases in Figure 5, it is not possible to select a geometrical parameter to simultaneously achieve large negative GVD and zero TOD. Therefore, it seems clear that the structure for soliton generation in standard SOI rib waveguides could be obtained only choosing a best tradeoff, not optimal, between negative GVD values and reduced TOD effect.

In addition to the before-mentioned potentials, the Si-nc slot waveguides present another very important advantage with respect to standard SOI rib waveguides. In fact, they can also strongly enhance the nonlinear parameter $\gamma$ as a result of the combination of very small effective mode area (in the slot region) and very large value of Kerr coefficient in

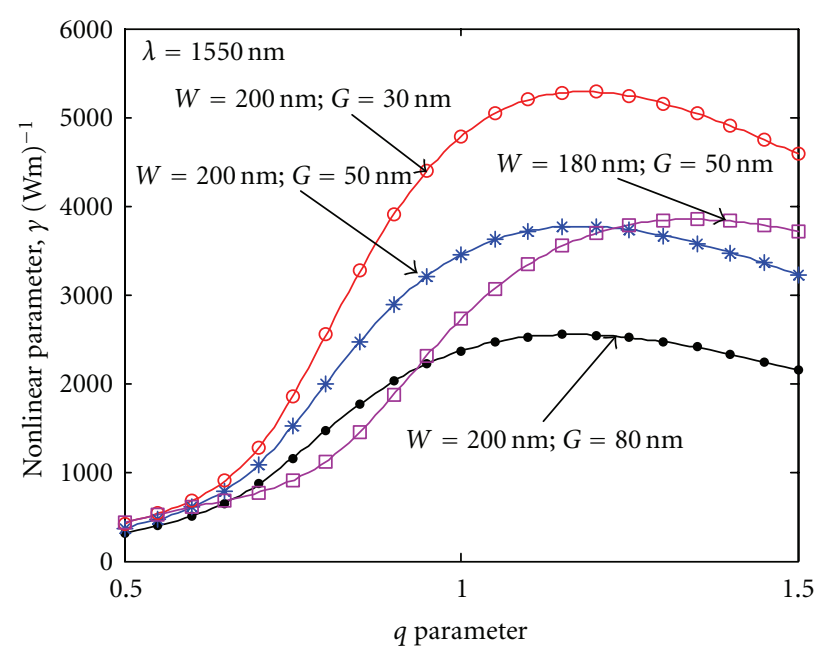

FIGURE 8: Nonlinear parameter versus $q$ for different values of $W$ and $G(\lambda=1550 \mathrm{~nm})$.

TABLE 1: Optical parameters of SOI rib waveguides.

\begin{tabular}{lccc}
\hline Parameter & $\begin{array}{c}\text { RIB no. 1 } \\
{[26]}\end{array}$ & $\begin{array}{c}\text { RIB no. 2 } \\
\text { This paper }\end{array}$ & $\begin{array}{c}\text { RIB no. } \\
\text { This paper }\end{array}$ \\
\hline Rib width $(\mathrm{nm})$ & 860 & 400 & 400 \\
Total rib height $(\mathrm{nm})$ & $\begin{array}{c}400 \\
(r=0.33)\end{array}$ & $\begin{array}{c}400 \\
(r=0.15)\end{array}$ & $\begin{array}{c}400 \\
(r=0.3)\end{array}$ \\
$\begin{array}{l}\text { Polarization state } \\
\text { Effective mode area } A_{\text {eff }}\end{array}$ & 0.13 & 0.23 & 0.28 \\
$\left(\mu \mathrm{m}^{2}\right)$ & 187.1 & 105.7 & 86.9 \\
$\begin{array}{l}\text { Nonlinear parameter } \gamma \\
\left((\mathrm{Wm})^{-1}\right)\end{array}$ & -2.26 & -3.5 & -2.5 \\
$\begin{array}{l}\mathrm{GVD} \text { coefficient } \beta_{2} \\
\left(\mathrm{ps}^{2} / \mathrm{m}\right)\end{array}$ & quasi-TM & quasi-TM \\
\hline
\end{tabular}

Three different SOI rib waveguides indicated as RIB no. 1, RIB no. 2, and RIB no. 3, have been considered.

silicon nanocrystals, $n_{2}=4 \times 10^{-17} \mathrm{~m}^{2} / \mathrm{W}$ [28]. These very large values of $y$ are shown in Figure 8, where the nonlinear parameter is plotted as a function of $q$ parameter for different values of $G$.

The plots show a $\gamma$ decrease with increasing $G$, for a fixed value of $W$. On the contrary, the nonlinear parameter decreases with decreasing $W$ for a given $G$. In addition, each curve shows a maximum point with a position independent from $G$ and corresponding to $q$ values close to 1.15 and 1.34 for $W=200 \mathrm{~nm}$ and $180 \mathrm{~nm}$, respectively. The effective mode area has been evaluated by means of the following relationship:

$$
A_{\text {eff }}^{-1}=\frac{\iint|F(x, y)|^{2}|F(x, y)|^{2} d x d y}{\iint|F(x, y)|^{2} d x d y \iint|F(x, y)|^{2} d x d y},
$$

being $F(x, y)$ the optical mode distribution in the waveguide cross section. For comparison purposes, the characteristics of some standard SOI rib waveguides are summarized in Table 1 in terms of their linear and nonlinear parameters and compared with data in the literature. 


\section{Soliton Theory}

The space-time evolution of optical solitons is governed by the pulse propagation equation written for the slowly varying amplitude $A(z, t)$ of the modal electric field $E(\mathbf{r}, t)$ traveling along the propagation direction $z$ [12]

$$
\begin{aligned}
\frac{\partial A}{\partial z}+ & \beta_{1} \frac{\partial A}{\partial t}+j \frac{1}{2} \beta_{2} \frac{\partial^{2} A}{\partial t^{2}}-\frac{1}{6} \beta_{3} \frac{\partial^{3} A}{\partial t^{3}} \\
= & -\frac{\left(\alpha^{(\mathrm{tot})}\right)}{2} A-0.5\left(\frac{\beta_{\mathrm{eff}}^{\mathrm{TPA}}}{A_{\mathrm{eff}}}\right)|A|^{2} A+j \gamma A|A|^{2} \\
& +j \frac{2 \pi}{\lambda} \Delta n A-\frac{\gamma}{\omega} \frac{\partial\left(|A|^{2} A\right)}{\partial t},
\end{aligned}
$$

where $\alpha^{(\text {tot })}$ is the waveguide total loss and $\beta_{1}$ is the inverse of group velocity. In (3), translational invariance along the propagation direction is assumed, thus the variable separation principle is applicable in the form $E(x, y, z, t)=$ $C \cdot F(x, y) A(z, t) e^{j \beta_{0} z} e^{-j \omega t}$, where $\beta_{0}$ is the propagation constant, $\omega$ is the angular frequency of the beam, and $C$ is a normalization constant [11]. The term $\Delta n$ is the effective index change due to plasma dispersion effect as induced by free carriers in the guiding structure.

The total loss coefficient in (3) is the sum of two contributions, $\alpha^{(\mathrm{tot})}=\alpha^{(\mathrm{prop})}+\alpha^{(\mathrm{FCA})}$, where $\alpha^{(\mathrm{prop})}$ is the propagation loss coefficient in the Si-nc slot waveguide, depending on the material absorption and fabrication process. Moreover, $\alpha^{(\mathrm{FCA})}$ is the contribution due to FCA, as induced by the change of the free carriers generated mainly by TPA of the input pulse. However, some additional comments are needed about the losses inside the Si-nc slot waveguides. In fact, a correct estimation of the propagation loss can be done by means of the following relationship [33]:

$$
\alpha^{(\text {prop })}=\Gamma_{\mathrm{Si}} \alpha_{\mathrm{Si}}+\Gamma_{\mathrm{Si}-\mathrm{nc}} \alpha_{\mathrm{Si}-\mathrm{nc}},
$$

being $\alpha_{\mathrm{Si}}$ and $\alpha_{\mathrm{Si}-\mathrm{nc}}$ the loss coefficients and $\Gamma_{\mathrm{Si}}$ and $\Gamma_{\mathrm{Si}-\mathrm{nc}}$ the power fractions in silicon and silicon nano-crystals, respectively,

$$
\Gamma_{\mathrm{Si}}=\frac{\iint_{\text {Silicon layers }}|\mathbf{E}|^{2} d x d y}{\iint|\mathbf{E}|^{2} d x d y}, \quad \Gamma_{\text {Si-nc }}=\frac{\iint_{\text {Slot }}|\mathbf{E}|^{2} d x d y}{\iint|\mathbf{E}|^{2} d x d y} .
$$

The term $\alpha^{(\mathrm{FCA})}$ depends on the contributions of FCA effect induced inside the silicon layers (being $\alpha_{\mathrm{Si}}^{(\mathrm{FCA})}$ the relevant coefficient), as well as that inside the slot region filled with Si-nc $\left(\alpha_{\text {Si-nc }}^{(\mathrm{FCA})}\right)$. It holds for silicon [34]

$$
\begin{aligned}
\alpha_{i}^{(\mathrm{FCA})}= & 8.5 \cdot 10^{-18} \cdot\left(\frac{\lambda_{i}}{1.55}\right)^{2} \Delta N_{e} \\
& +6.0 \cdot 10^{-18} \cdot\left(\frac{\lambda_{i}}{1.55}\right)^{2} \Delta N_{h} \\
= & \sigma_{i} \cdot N_{c}=\sigma_{0} \cdot\left(\frac{\lambda_{i}}{1.55}\right)^{2} N_{c},
\end{aligned}
$$

where $N_{c}=\Delta N_{e}=\Delta N_{h}$ is the density of electron-hole pairs generated by TPA process inside the silicon layers. The coefficient $\sigma_{0}=1.45 \times 10^{-17} \mathrm{~cm}^{-2}$ [35] is the FCA crosssection measured at $\lambda=1550 \mathrm{~nm}$ and $\lambda_{i}$ is the relevant mode wavelength. More difficult is the evaluation of $\alpha_{\mathrm{Si}-\mathrm{nc})}^{(\mathrm{FCA})}$. As described in [29], the pump-induced FCA loss in Si-nc material is related to the excited population of nanocrystals by

$$
\alpha_{\mathrm{Si}-\mathrm{nc}}^{(\mathrm{FCA})}=\sigma_{\mathrm{Si}-\mathrm{nc}} N_{\mathrm{exc}},
$$

where $\sigma_{\mathrm{Si}-\mathrm{nc}}$ is the FCA cross-section; that is, $2.6 \times 10^{-17} \mathrm{~cm}^{-2}$ [29]. The term $N_{\text {exc }}$ is the number of excited nanocrystals. Now, the carrier dynamics inside the silicon nanocrystals can be described by means of a four level system, where $N_{\text {exc }}$ represents the population density of the second energy level. The decay lifetimes $\left(\tau_{1}, \tau_{3}\right)$ can be estimated as $1 \mathrm{ps}$ for levels 1 and 3 so that these levels are almost always empty. Differently, the lifetime $\tau_{2}$ estimated for level 2 is $30 \mu \mathrm{s}$. However, the model in this section does not allow the rigorous evaluation of $N_{c}$ and $N_{\text {exc }}$, since it does not include the time dynamics of four level energy system related to the nanocrystals. However, the main goal of the equations proposed in this section is to give an engineering estimation of the influence of both TPA and FCA effects on the formation of optical solitons inside Si-nc slot waveguides. Thus, under this point of view, we conjecture to estimate $N_{c}$ and $N_{\text {exc }}$ as $N_{c} \approx N \Gamma_{\mathrm{Si}}$ and $N_{\text {exc }} \approx N \Gamma_{\text {Si-nc }}$, being $N$ the solution of the following rate equation:

$$
\frac{d N}{d t}=-\frac{N}{\bar{\tau}}+\frac{\beta_{\mathrm{eff}}^{\mathrm{TPA}}}{2 \hbar \omega}\left(\frac{|A(z, t)|^{2}}{A_{\mathrm{eff}}}\right)^{2} .
$$

In (8), two semiempirical parameters have been introduced, namely $\bar{\tau}$ as an effective recombination lifetime and $\beta_{\text {eff }}^{\text {TPA }}$ as an effective TPA coefficient. Both these values could be appropriately selected, according to experimental data. However, we have estimated $\beta_{\mathrm{eff}}^{\text {TPA }}$ as

$$
\beta_{\text {eff }}^{\mathrm{TPA}}=\Gamma_{\mathrm{Si}} \beta_{\mathrm{Si}}^{\mathrm{TPA}}+\Gamma_{\mathrm{Si}-\mathrm{nc}} \beta_{\mathrm{Si}-\mathrm{nc}}^{\mathrm{TPA}},
$$

being $\beta_{\mathrm{Si}}^{\mathrm{TPA}}=0.5 \mathrm{~cm} / \mathrm{GW}$ [35] and $\beta_{\mathrm{Si}-\mathrm{nc}}^{\mathrm{TPA}}=5 \mathrm{~cm} / \mathrm{GW}$ [28] for silicon and Si-nc layers, respectively.

In conclusion, (3)-(9) lead to numerically investigate the formation of optical solitons inside Si-nc slot waveguides through a semi-analytical model. Hereinafter, normalized variables are used, as $U(z, t)=A(z, t) / \sqrt{P_{0}}$ and $\tau=(t-$ $\left.z \cdot \beta_{1}\right) / T_{0}$, where $P_{0}$ is the peak power of input Gaussian pulse and $T_{0}$ is related to the Gaussian pulse FWHM width by $T_{0}=T_{\mathrm{FWHM}} / 1.665$.

To numerically solve the coupled system (3)-(9), the collocation method [36] has been used by developing the unknown functions (i.e., $A, N$ ) as a linear combination of $M$ orthogonal functions $\phi_{m}(\tau)$ through $M$ weight functions $c_{m}(z)$, as

$$
X(z, \tau)=\sum_{m=1}^{M} c_{m}(z) \phi_{m}(\tau)
$$


TABLE 2: Slot structure optical parameters at $\lambda=1550 \mathrm{~nm}$.

\begin{tabular}{lccc}
\hline Parameter & SLOT\#1 & SLOT\#2 & SLOT\#3 \\
\hline$W(\mathrm{~nm})$ & 180 & 200 & 200 \\
Total height $(\mathrm{nm})$ & 440 & 442 & 422 \\
Polarization & quasi-TM & quasi-TM & quasi-TM \\
$G(\mathrm{~nm})$ & 50 & 50 & 30 \\
$q$ & 1.08 & 0.98 & 0.98 \\
$A_{\text {eff }}\left(\mu \mathrm{m}^{2}\right)$ & 0.04881 & 0.0485 & 0.0347 \\
$n_{\text {eff }}$ & 1.446 & 1.566 & 1.673 \\
$\Gamma_{\mathrm{Si}}$ & 0.16 & 0.1716 & 0.2201 \\
$\Gamma_{\mathrm{Si}-\mathrm{nc}}$ & 0.30 & 0.3326 & 0.2937 \\
$\beta_{2}\left(\mathrm{ps}^{2} / \mathrm{m}\right)$ & -4.074 & -1.052 & -2.76 \\
$\gamma\left((\mathrm{Wm})^{-1}\right)$ & 3255 & 3385 & 4602 \\
\hline
\end{tabular}

where $X(z, \tau)$ states for each unknown function. HermiteGauss functions have been chosen as basis functions that is, $\phi_{m}(\tau)=H_{m-1}(\tau) \exp \left(-0.5 \tau^{2}\right)$. The unknown coefficients $c_{m}(z)$ are determined by satisfying the differential equation system at $M$ collocation points $\tau_{m}$, such that $H_{M}\left(\tau_{m}\right)=0$, $m=1, \ldots, M$. Thus, with at least $M=35$ collocation points, the system of partial differential (3)-(9) in $z, \tau$ domain becomes a system of ordinary differential matrix equations in $z$, which can be solved by a fourth-order RungeKutta algorithm. The overlap integrals have been carefully evaluated again through the full vectorial FEM.

In the following simulations, we have considered the Si-nc slot waveguide excited by a Gaussian optical pulse with $T_{\mathrm{FWHM}}=116 \mathrm{fs}$, and peak power selected to satisfy a specific condition, $N=\left(L_{D} / L_{\mathrm{NL}}\right)^{1 / 2}=1.7$. An effective lifetime $\bar{\tau}=0.1 \mathrm{~ns}$ is assumed, which is supposed to be a conservative choice although one order of magnitude less than the effective recombination lifetime in standard SOI rib waveguides, $\tau_{\text {eff }}=1 \mathrm{~ns}$. In fact, the introduction of nanometre defects in the silicon waveguide (nanocrystals) is expected to shorten the recombination time to a few of tens of picoseconds, while simultaneously increasing the light scattering, that is, the optical losses [37-39]. However, since the dispersion effects (GVD, TOD) take place in the subpicosecond regime $(<1 \mathrm{ps})$ as well as FCA induced by the TPA produces negligible effects for short pulses, the effective recombination lifetime $\bar{\tau}$ should play a secondary rule in the optical soliton generation. Finally, we have assumed $\alpha_{\mathrm{Si}}=1 \mathrm{~dB} / \mathrm{cm}$ and $\alpha_{\mathrm{Si}-\mathrm{nc}}=15 \mathrm{~dB} / \mathrm{cm}$ as average values between those indicated in the literature $[27,28]$. Other optical parameters are summarized in Table 2 for Si-nc slot structures, named as SLOT no. 1, SLOT no. 2, and SLOT no. 3 .

It is worth to outline that the Si-nc slot structures used in our theoretical investigation are very similar to those proposed in some experimental works [27-29]. In particular, the same properties and fabrication procedure are assumed here. Thus, our structures could be fabricated starting from a SOI substrate and should include successive plasma-enhanced chemical-vapor deposition (PECVD) of a crystalline silicon layer, a Si: nc layer and an amorphous silicon layer on the

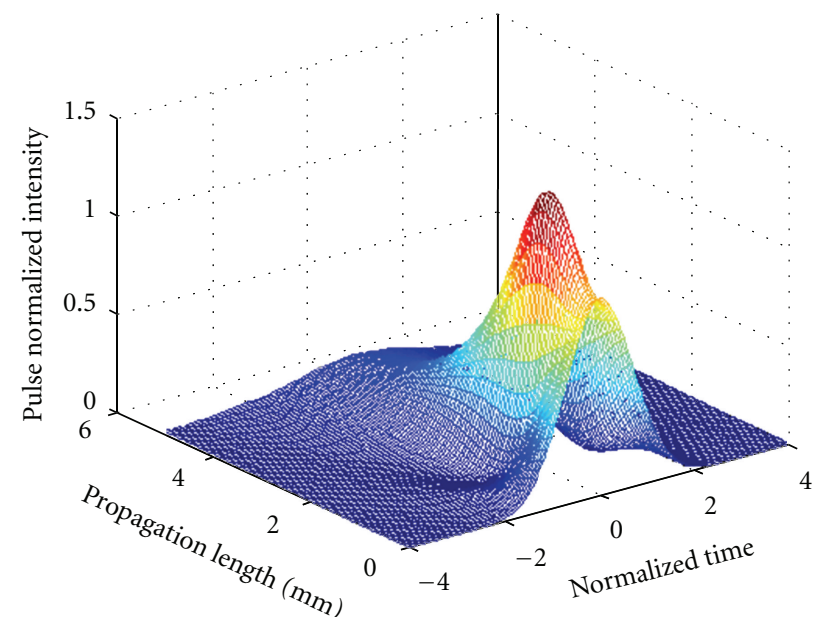

Figure 9: Space-time evolution of the Gaussian pulse into an optical soliton in slot waveguide SLOT no. 1.

top. Final result should be characterized by $\mathrm{Si}: \mathrm{nc}$ with 0.08 silicon excess, for an annealing temperature of $800^{\circ} \mathrm{C}$ [28].

Figure 9 shows that the space-time evolution of input Gaussian pulse can generate an optical soliton in structure SLOT no. 1.

In fact, as the pulse propagates along the Si-nc slot waveguide, it converts its shape into a hyperbolic-secant pulse, starting from the Gaussian profile. In particular, after a short propagation length, less than $1 \mathrm{~mm}$, the optical pulse is affected by a temporal broadening and distortion, but, at around $1.2 \mathrm{~mm}$, the pulse recovers and converts itself in a compressed hyperbolic-secant shape. This pattern results to be rigorously periodic. It is worth to remind that the waveguide SLOT no. 1 has been designed to have at the same time large negative $\beta_{2}$ and $\beta_{3} \approx 0$ according with Figures 5 and 6 . However, the presence of optical losses, TPA and FCA is expected to enable a stable soliton for lengths larger than $3 \mathrm{~mm}$. Thus, the plot in Figure 9 clearly shows as it is possible the formation of an optical soliton inside a very short Si-nc slot waveguide (only $1.2 \mathrm{~mm}$ long).

Figure 10 shows the soliton formation inside the three investigated Si-nc slot waveguides. For comparison, the optical soliton excited in the SOI rib waveguide proposed in [26] is sketched, too. The plot evidences how the input Gaussian pulse converts itself as an ultrafast soliton (FWHM width of about $60 \mathrm{fs}$ ) inside the Si-nc slot waveguide, having a typical sech-like profile after a propagation length depending on the specific structure. In fact, in case of SLOT no. 1 or SLOT no. 3, the soliton formation is achieved at $z=1.2$ and $1.8 \mathrm{~mm}$, that is, well shorter lengths than in rib SOI rib waveguide [26], or in Si-nc slot waveguide named SLOT no. 2 , where the optical soliton still appears at about $z \cong 5 \mathrm{~mm}$.

This means that thin Si-nc layers are required $(W \times$ $G=9000 \mathrm{~nm}^{2}$ and $W \times G=6000 \mathrm{~nm}^{2}$ in SLOT no. 1 and SLOT no. 3, resp.) to maximize the complex effect interplay generating a soliton over very short guiding structure lengths, while larger Si-nc cross-sections $(W \times G=$ $10000 \mathrm{~nm}^{2}$ in SLOT no. 2) are not convenient over standard rib structures without silicon nanocrystals. 


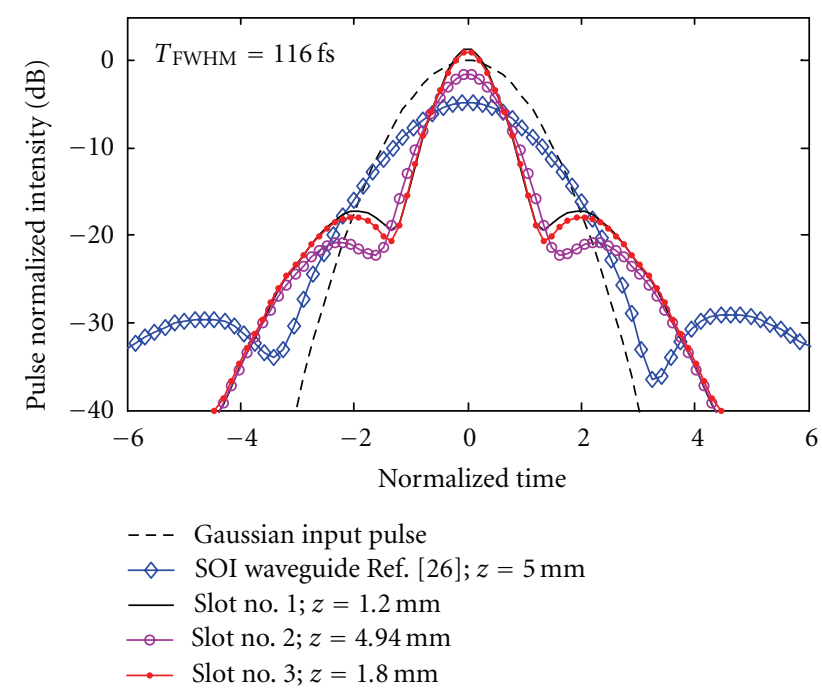

FIGURE 10: Temporal evolution of optical solitons in different Si-nc slot waveguides and comparison with literature.

\section{Conclusions}

In this paper, we have theoretically demonstrated for the first time the formation of optical solitons inside Si-nc slot waveguides on SOI platform. Design guidelines of these structures are presented in order to reduce the device lengths for generating ultrafast solitons. Results prove that optimal waveguides can induce optical soliton formation over a propagation length 3 times less than in standard SOI rib waveguides. The potential of Si-nc slot waveguides in SOI technology is very significant, and it could represent a very promising technology for ultrafast nonlinear optical processing and fast telecommunications systems.

\section{References}

[1] R. Soref, "The past, present, and future of silicon photonics," IEEE Journal on Selected Topics in Quantum Electronics, vol. 12, no. 6, pp. 1678-1687, 2006.

[2] G. T. Reed, "The optical age of silicon," Nature, vol. 427, no. 6975, pp. 595-596, 2004.

[3] G. T. Reed and A. P. Knights, Silicon Photonics: An Introduction, John Wiley, West Sussex, UK, 2004.

[4] L. Liao, A. Liu, D. Rubin et al., " 40 Gbit/s silicon optical modulator for high-speed applications," Electronics Letters, vol. 43, no. 22, pp. 1196-1197, 2007.

[5] R. Claps, D. Dimitropoulos, Y. Han, and B. Jalali, "Observation of Raman emission in silicon waveguides at $1.54 \mu \mathrm{m}$," Optics Express, vol. 10, no. 22, pp. 1305-1313, 2002.

[6] R. Claps, D. Dimitropoulos, V. Raghunathan, Y. Han, and B. Jalali, "Observation of stimulated Raman amplification in silicon waveguides," Optics Express, vol. 11, no. 15, pp. 17311739, 2003.

[7] A. Liu, H. Rong, M. Paniccia, O. Cohen, and D. Hak, "Net optical gain in a low loss silicon-on-insulator waveguide by stimulated Raman scattering," Optics Express, vol. 12, no. 18, pp. 4261-4268, 2004.
[8] Q. Xu, V. R. Almeida, and M. Lipson, "Time-resolved study of Raman gain in highly confined silicon-on-insulator waveguides," Optics Express, vol. 12, no. 19, pp. 4437-4442, 2004.

[9] Q. Xu, V. R. Almeida, and M. Lipson, "Demonstration of high Raman gain in a submicrometer-size silicon-on-insulator waveguide," Optics Letters, vol. 30, no. 1, pp. 35-37, 2005.

[10] R. L. Espinola, J. I. Dadap, R. M. Osgood Jr., S. J. McNab, and Y. A. Vlasov, "Raman amplification in ultrasmall silicon-oninsulator wire waveguides," Optics Express, vol. 12, no. 16, pp. 3713-3718, 2004.

[11] V. M. N. Passaro and F. De Leonardis, "Space-Time modeling of Raman pulses in silicon-on-insulator optical waveguides," Journal of Lightwave Technology, vol. 24, no. 7, pp. 2920-2931, 2006.

[12] F. De Leonardis and V. M. N. Passaro, "Ultrafast Raman pulses in SOI optical waveguides for nonlinear signal processing," IEEE Journal on Selected Topics in Quantum Electronics, vol. 14, no. 3, Article ID 4538023, pp. 739-751, 2008.

[13] O. Boyraz and B. Jalali, "Demonstration of a silicon Raman laser," Optics Express, vol. 12, no. 21, pp. 5269-5273, 2004.

[14] M. Krause, H. Renner, and E. Brinkmeyer, "Analysis of Raman lasing characteristics in silicon-on-insulator waveguides," Optics Express, vol. 12, no. 23, pp. 5703-5710, 2004.

[15] H. Rong, R. Jones, A. Liu et al., "A continuous-wave Raman silicon laser," Nature, vol. 433, no. 7027, pp. 725-728, 2005.

[16] H. Rong, A. Liu, R. Jones et al., "An all-silicon Raman laser," Nature, vol. 433, no. 7023, pp. 292-294, 2005.

[17] O. Boyraz and B. Jalali, "Demonstration of directly modulated silicon Raman laser,” Optics Express, vol. 13, no. 3, pp. 796800, 2005.

[18] F. De Leonardis and V. M. N. Passaro, "Modelling of Raman amplification in silicon-on-insulator optical microcavities," New Journal of Physics, vol. 9, article A25, pp. 1-24, 2007.

[19] H. Rong, S. Xu, O. Cohen et al., "A cascaded silicon Raman laser," Nature Photonics, vol. 2, no. 3, pp. 170-174, 2008.

[20] V. M. N. Passaro and F. de Leonardis, "Investigation of SOI raman lasers for mid-infrared gas sensing," Sensors, vol. 9, no. 10, pp. 7814-7836, 2009.

[21] H. Rong, Y. H. Kuo, A. Liu, M. Paniccia, and O. Cohen, "High efficiency wavelength conversion of $10 \mathrm{~Gb} / \mathrm{s}$ data in silicon waveguides," Optics Express, vol. 14, no. 3, pp. 1182-1188, 2006.

[22] Y. H. Kuo, H. Rong, V. Sih, S. Xu, M. Paniccia, and O. Cohen, "Demonstration of wavelength conversion at $40 \mathrm{~Gb} / \mathrm{s}$ data rate in silicon waveguides," Optics Express, vol. 14, no. 24, pp. 11721-11726, 2006.

[23] V. G. Ta'eed, M. D. Pelusi, B. J. Eggleton et al., "Broadband wavelength conversion at $40 \mathrm{~Gb} / \mathrm{s}$ using long serpentine $\mathrm{As}_{(2)} \mathrm{S}_{3}$ planar waveguides," Optics Express, vol. 15, no. 23, pp. 1504715052, 2007.

[24] B. G. Lee, A. Biberman, A. C. Turner-Foster et al., "Demonstration of broadband wavelength conversion at $40 \mathrm{~Gb} / \mathrm{s}$ in silicon waveguides," IEEE Photonics Technology Letters, vol. 21, no. 3, pp. 182-184, 2009.

[25] G. P. Agrawal, Nonlinear Fiber Optics, Academic Press, London, UK, 3rd edition, 2001.

[26] J. Zhang, Q. Lin, G. Piredda, R. W. Boyd, G. P. Agrawal, and P. M. Fauchet, "Optical solitons in a silicon waveguide," Optics Express, vol. 15, no. 12, pp. 7682-7688, 2007.

[27] J. Blasco, J. V. Galán, P. Sanchis et al., "FWM in silicon nanocrystal-based sandwiched slot waveguides," Optics Communications, vol. 283, no. 3, pp. 435-437, 2010. 
[28] A. Martínez, J. Blasco, P. Sanchis et al., "Ultrafast all-optical switching in a silicon-nanocrystal-based silicon slot waveguide at telecom wavelengths," Nano Letters, vol. 10, no. 6, pp. 15061511, 2010.

[29] T. Creazzo, B. Redding, E. Marchena, S. Shi, and D. W. Prather, "Free-carrier absorption modulation in silicon nanocrystal slot waveguides," Optics Letters, vol. 35, no. 21, pp. 3691-3693, 2010.

[30] L. Zhang, Y. Yue, Y. Xiao-Li, J. Wang, R. G. Beausoleil, and A. E. Willner, "Flat and low dispersion in highly nonlinear slot waveguides," Optics Express, vol. 18, no. 12, pp. 13187-13193, 2010.

[31] L. Zhang, Y. Yue, R. G. Beausoleil, and A. E. Willner, "Flattened dispersion in silicon slot waveguides," Optics Express, vol. 18, no. 19, pp. 20529-20534, 2010.

[32] Comsol Multiphysics by COMSOL, Stockholm, single license, 2005.

[33] R. A. Soref, S. J. Emelett, and W. R. Buchwald, "Silicon waveguided components for the long-wave infrared region," Journal of Optics A, vol. 8, no. 10, pp. 840-848, 2006.

[34] R. A. Soref and B. R. Bennett, "Electrooptical effects in silicon," IEEE Journal of Quantum Electronics, vol. QE-23, no. 1, pp. 123-129, 1987.

[35] R. Claps, V. Raghunathan, D. Dimitropoulos, and B. Jalali, "Influence of nonlinear absorption on Raman amplification in Silicon waveguides," Optics Express, vol. 12, no. 12, pp. 27742780, 2004.

[36] S. Deb and A. Sharma, "Nonlinear pulse propagation through optical fibers: an efficient numerical method," Optical Engineering, vol. 32, pp. 695-699, 1993.

[37] T. Tanabe, K. Nishiguchi, A. Shinya et al., "Fast alloptical switching using ion-implanted silicon photonic crystal nanocavities," Applied Physics Letters, vol. 90, no. 3, Article ID 031115, 2007.

[38] M. Waldow, T. Plötzing, M. Gottheil et al., "25ps all-optical switching in oxygen implanted silicon-on-insulator microring resonator," Optics Express, vol. 16, no. 11, pp. 7693-7702, 2008.

[39] K. Preston, P. Dong, B. Schmidt, and M. Lipson, "High-speed all-optical modulation using polycrystalline silicon microring resonators," Applied Physics Letters, vol. 92, no. 15, Article ID 151104,2008 

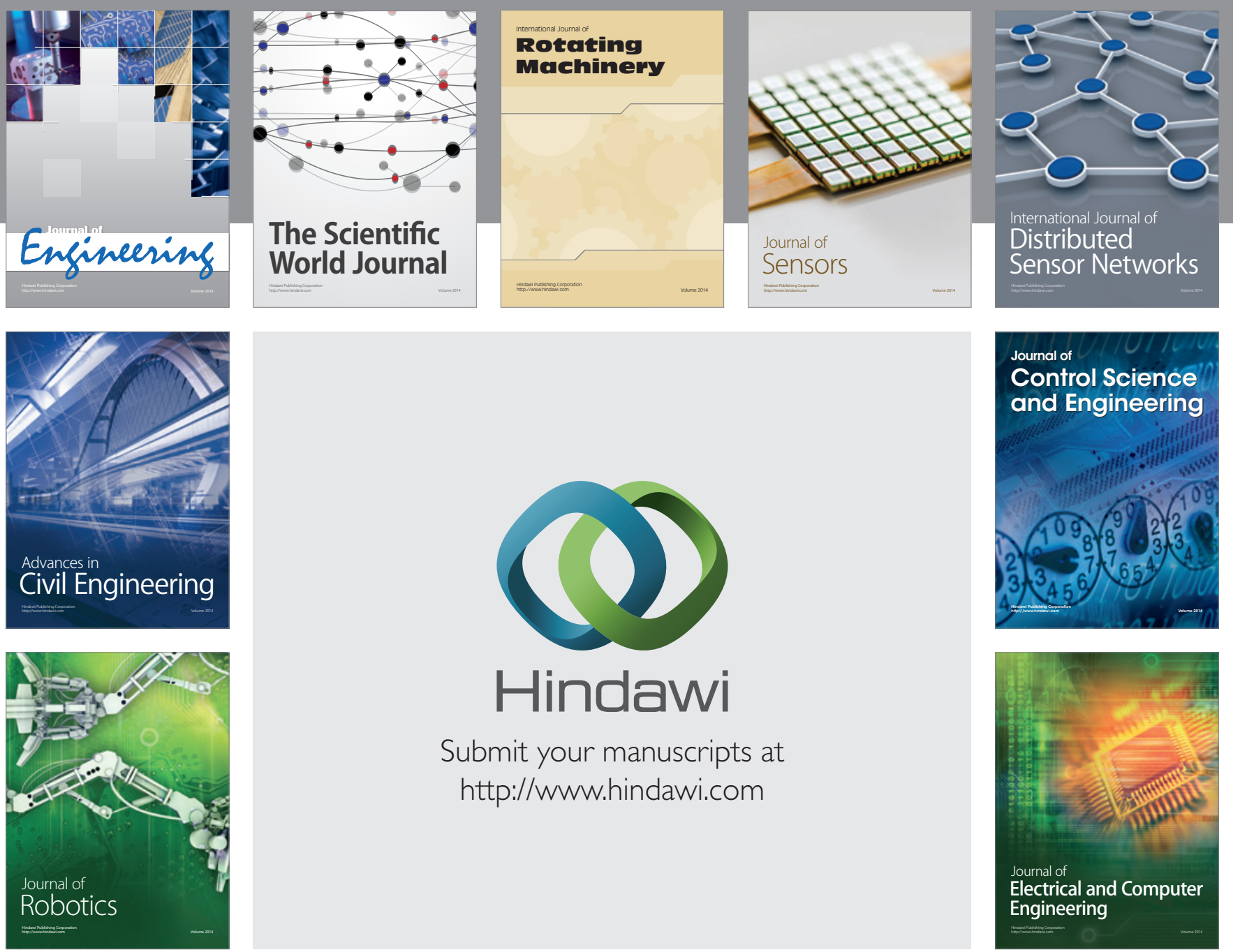

Submit your manuscripts at

http://www.hindawi.com
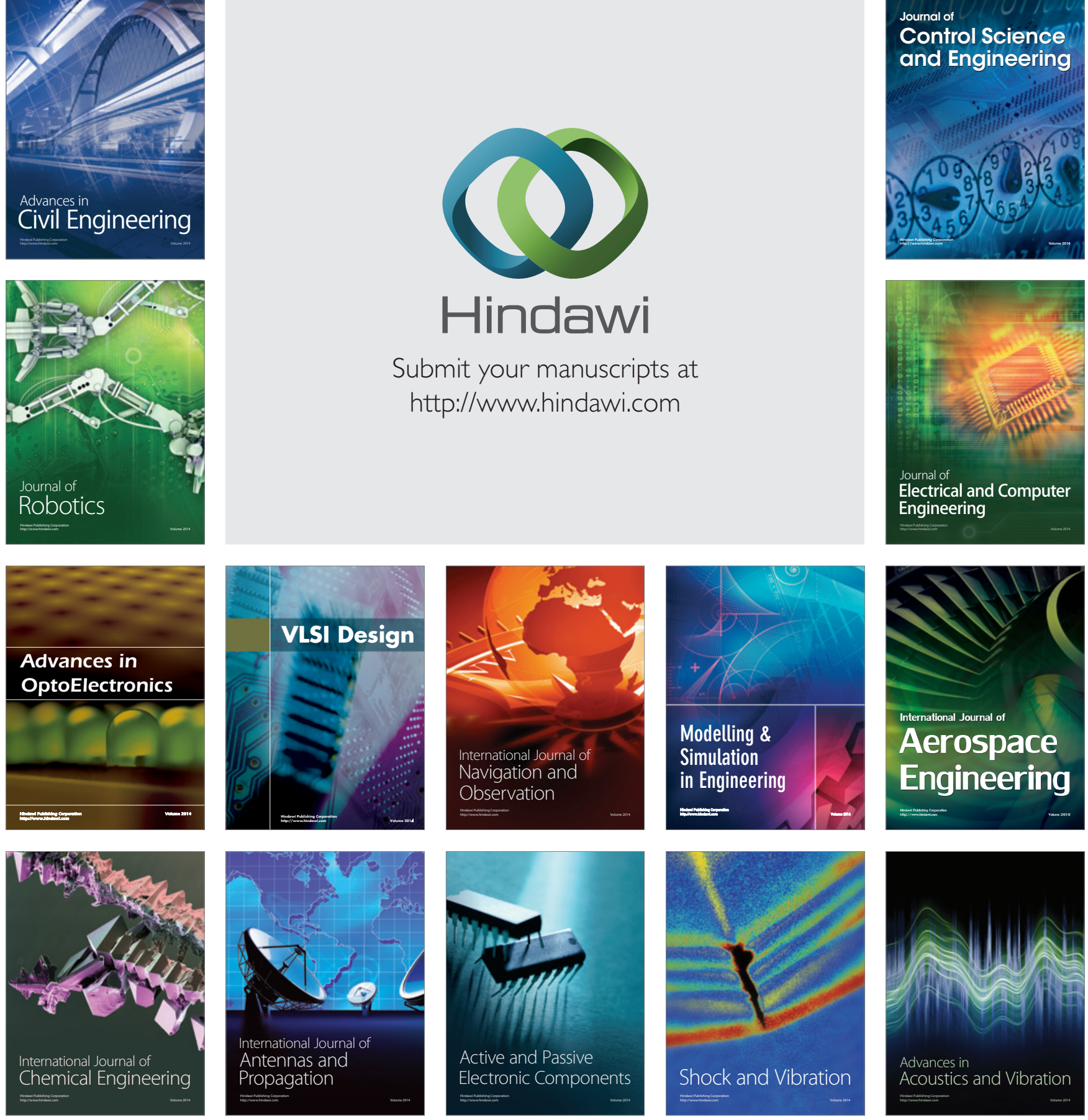